\title{
Physical activity level of adult cats with varied feeding frequency
}

\author{
Ping Deng ${ }^{1}$, Ryan W. Grant ${ }^{2}$ and Kelly S. Swanson ${ }^{1,2,3 *}$ \\ ${ }^{1}$ Department of Animal Sciences, University of Illinois, 162 Animal Sciences Laboratory, 1207 West Gregory Drive, \\ Urbana, IL 61801, USA \\ ${ }^{2}$ Division of Nutritional Sciences, University of Illinois, Urbana, IL, USA \\ ${ }^{3}$ Department of Veterinary Clinical Medicine, University of Illinois, Urbana, IL, USA
}

(Received 13 October 2010 - Revised 26 January 2011 - Accepted 13 February 2011)

\section{Abstract}

The prevalence of feline obesity is influenced by numerous factors, including inactivity and overconsumption of food. The objective of the present study was to evaluate the effect of feeding frequency on physical activity in adult cats. A total of twelve healthy adult cats were used in a cross-over study consisting of $32 \mathrm{~d}$. In each of the two periods, six cats were fed either two meals or four meals daily. Throughout the study, cats were fed the same diet at amounts to maintain body weight and body condition score. Cats were individually housed $4 \mathrm{~h} / \mathrm{d}$ at each scheduled feeding time, while for the other $20 \mathrm{~h}$, cats were group-housed to allow for voluntary physical activity in the room with a $16 \mathrm{~h}$ light $-8 \mathrm{~h}$ dark cycle. Voluntary activity levels were evaluated using Actical activity collars for seven consecutive days in each period. Daily average activity level for two-meal-fed cats (20.04 (SEM 2.19), activity counts/epoch (15 s)) was not different from four-meal-fed cats (20.14 (SEM 2.15), activity counts/epoch (15s); P>0.05). In conclusion, when group-housed cats are fed to maintain their body weight and body condition score, varied feeding frequency between twice and four times daily may not affect activity levels.

\section{Key words: Obesity: Cats: Feeding frequency: Physical activity: Activity pattern}

The prevalence of feline obesity has rapidly increased over recent decades. One of the most recent studies has suggested that approximately $35 \%$ of adult cats in the USA were considered either overweight or obese ${ }^{(1)}$. Obesity prevalence is influenced by numerous factors. Activity is one of the factors influenced by both individual (e.g. neuter) and environmental variables (e.g. accommodations, owner behaviours and tendencies). Decreased physical activity has been identified as a major risk factor for the development of obesity and feline diabetes $^{(2)}$. However, strategies to increase physical activity to reduce obesity incidence have been lacking. Increasing feeding frequency has been suggested as a contributing factor to manage body weight $(\mathrm{BW})^{(3)}$. It was our hypothesis that by increasing feeding frequency, without altering the amount fed per day, more common feelings of hunger and the consequent search for food would alter activity pattern and increase overall activity level. The effect of feeding frequency on physical activity level has not been quantitatively measured in cats. Moreover, it is not clear how increased feeding frequency may affect $24 \mathrm{~h}$ activity patterns. The use of activity monitors allowed us to continuously monitor activity during several days in the dark and light periods without human interference, which has been demonstrated to be an accurate method of measuring physical activity in cats ${ }^{(4,5)}$. Therefore, the objective of the present study was to evaluate the effect of feeding frequency on physical activity in adult cats.

\section{Materials and methods}

\section{Animals and diets}

A total of twelve healthy adult, neutered, male domestic shorthair cats (approximately 3 years old; 4.74 (SEM0.16) $\mathrm{kg}$ BW; $4 \cdot 5-5 \cdot 5$ body condition score (BCS) on a 9-point scale) were used in this experiment. Cats were group-housed in the same room $\left(26.67 \mathrm{~m}^{2} \times 3 \mathrm{~m}\right)$ most of the day but were individually housed $4 \mathrm{~h}$ per day in stainless-steel cages $(0.61 \mathrm{~m} \times 0.61 \mathrm{~m} \times 0.61 \mathrm{~m})$ in the animal facility of the Edward R. Madigan Laboratory at the University of Illinois (Urbana, IL, USA). The room was environmentally controlled $\left(20^{\circ} \mathrm{C}\right)$ with a $16 \mathrm{~h}$ light $-8 \mathrm{~h}$ dark cycle. The $16 \mathrm{~h}$ light period lasted from 06.00 to 22.00 hours each day. Cats were allowed access to various toys and scratching poles for behavioural enrichment and to encourage play.

Cats were fed the same dry kibble diet at amounts to maintain BW and BCS throughout the study. Before the present study, cats were fed commercial or experimental diets once

Abbreviations: BW, body weight; BCS, body condition score.

*Corresponding author: K. S. Swanson, fax +1 217333 7861, email ksswanso@illinois.edu 
daily in the morning (08.00-09.00 hours). The experimental diet fed in the present study was based on maize gluten meal, brewer's rice, wheat flour and poultry by-product meal, and was formulated to contain moderate concentrations of carbohydrate, fat and protein (approximately 33\% of energy from each). The diet was formulated to meet all nutrient recommendations for adult cats as provided by the Association of American Feed Control Officials (Oxford, IN, USA) ${ }^{(6)}$. Water was available ad libitum throughout the experiment. All animal procedures were approved by the University of Illinois Institutional Animal Care and Use Committee before experimentation.

\section{Experimental design}

A cross-over study design consisting of $32 \mathrm{~d}$ (two $16 \mathrm{~d}$ periods) was used. Voluntary activity levels were measured from the morning of the 8th day to the morning of the 15th day. In the first period, cats (six animals per treatment) were fed either two meals or four meals daily and vice versa for the second period. Half of the daily intake was offered to twomeal-fed cats at 08.00 and 20.00 hours. At 08.00, 12.00, 16.00 and 20.00 hours, one-quarter of the daily intake was offered to four-meal-fed cats, respectively. After the cross-over study, all twelve cats were changed back to their normal feeding pattern, during which all cats were fed once daily at 08.00 hours. Activity measurements were also performed during this time as in the previous two periods. Cats were maintained on a strict housing schedule, being individually housed in cages for access to diet from 08.00 to $09.00,12.00$ to $13.00,16.00$ to 17.00 , and 20.00 to 21.00 hours each day. For the other $20 \mathrm{~h}$, cats were group-housed in the room to allow for voluntary physical activity without human interference.

\section{Physical activity level assessment}

Voluntary activity levels were evaluated using Actical activity monitors (Mini Mitter, Bend, OR, USA), which were worn about the neck for seven consecutive days at the middle of each period as performed previously in research cats in our laboratory $^{(4,5)}$. The monitors contain omnidirectional sensors capable of accurately incorporating both intensity and duration of movement. The Actical software analysed the data compiled by the monitor and converted it into arbitrary numbers referred to as 'activity counts'. Activity levels were expressed as activity counts per epoch (epoch length $15 \mathrm{~s}$ ). Values represent the mean epoch activity count over $7 \mathrm{~d}$, during the selected hours (light hours, dark hours and average throughout the entire day) without human interference. Monitors were calibrated by Actical. To control for variability, cats wore the same monitor for each period of the study.

\section{Statistical analyses}

Data were analysed using the Mixed procedure of Statistical Analysis Systems 9.1 (SAS Institute Inc., Cary, NC, USA). Feeding frequency and time were tested as the main fixed effects. Random effects of cat, sequence and period were included in
Table 1. Physical activity levels of adult cats fed two or four meals daily (Mean values (activity counts) with their standard errors, $n 11$ (two meals) and 12 (four meals))

\begin{tabular}{lrrrrrrr}
\hline & \multicolumn{2}{c}{ Two meals } & & \multicolumn{2}{c}{ Four meals } \\
\cline { 2 - 3 } Items & Mean & SEM & & Mean & SEM & $P$ \\
\hline Daily activity levels & 20.04 & 2.19 & & 20.14 & 2.15 & 0.94 \\
Activity during the light period & 25.02 & 2.68 & & 24.86 & 2.63 & 0.93 \\
Activity during the dark period & 10.05 & 1.77 & & 10.71 & 1.75 & 0.52 \\
Activity ratio, light:dark & 2.79 & 0.37 & & 2.65 & 0.37 & 0.37
\end{tabular}

Data were compiled by activity collars by Actical software. Activity levels were defined as arbitrary units referred to 'activity counts'. Activity is expressed as activity counts per epoch (epoch length $15 \mathrm{~s}$ ).

the model. Results are presented as means with their standard errors. A probability of $P<0.05$ was considered significant.

\section{Results}

There were no differences $(P>0.05)$ in daily average activity, activity during the light period or activity during the dark period between two-meal-fed cats and four-meal-fed cats (Table 1). Daily activity levels of all twelve cats peaked during meal times, which were all during the light period. The daily average activity level for one-meal-fed cats $(15.57$ (sEm 2.03), activity counts/epoch (15s)) was numerically (22.5\%) lower than cats on the multiple-meal treatments, two-meal-fed treatment (20.04 (SEM 2.19), activity counts/ epoch (15s)) or four-meal-fed treatment (20·14 (SEM 2.15), activity counts/epoch (15s)). Because the once daily feeding was not included in the cross-over design, it is, however, not appropriate to analyse these differences statistically. As shown in Fig. 1, the daily activity pattern of one-meal-fed cats differed from multiple-meal-fed cats. Although activity during the dark period and 08.00 hours meal were similar for all cats, peaks of activity for one-meal-fed cats tended to occur during group-housing immediately following an hour in their individual cage rather than peaks during meal times for multiple-meal-fed cats.

\section{Discussion}

To our knowledge, this is the first study to quantitatively evaluate the physical activity level of adult cats with varied feeding frequency. All cats were fed the same dry kibble diet, consistent food intakes to maintain BW and BCS, and were kept under the same daily schedule (in and out of cages) to eliminate the effects of factors other than feeding frequency. The activity monitors have been demonstrated to be an accurate method of measuring physical activity in cats $^{(4,5)}$. The results showed that the daily average activity level for two-meal-fed cats was not different from four-mealfed cats. The feeding frequency had no effects on this crossover design. It is possible that the interactions between the cats from two different treatments attenuated the effects of feeding frequencies on the activity levels. Cats in the twicedaily-feeding group may have increased activity associated 


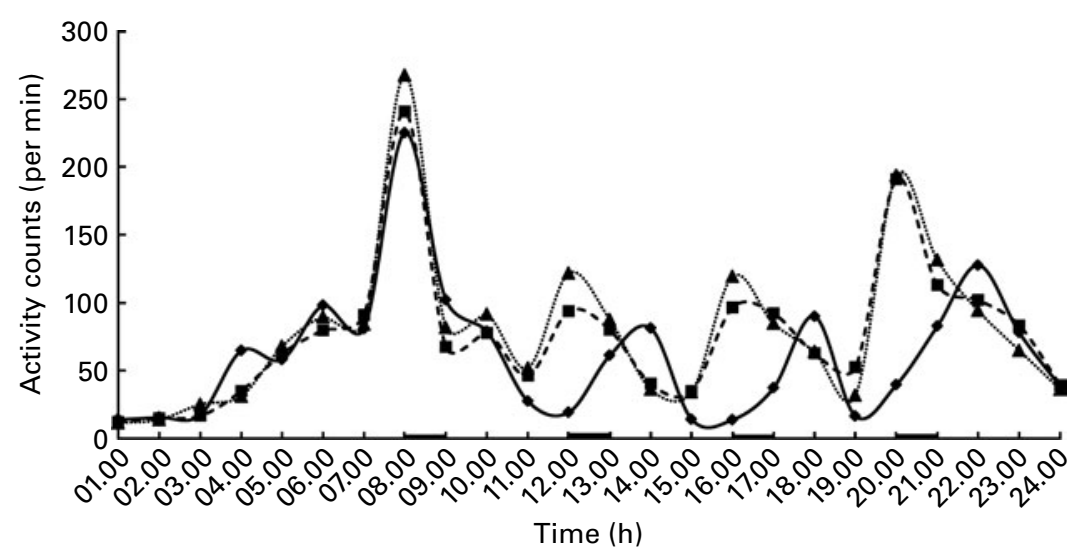

Fig. 1. Effects of feeding frequency on daily activity patterns in adult cats. A $16 \mathrm{~h}$ light-8h dark cycle was used in the animal facility. The $16 \mathrm{~h}$ light period lasted from 06.00 to 22.00 hours, and the $8 \mathrm{~h}$ dark period lasted from 22.00 to 06.00 hours. Cats were maintained on a strict housing schedule, being individually housed in cages for access to diet from 08.00 to $09.00,12.00$ to $13.00,16.00$ to 17.00 and 20.00 to 21.00 hours each day, which are shown as thick lines on the $x$-axis. For the other $20 \mathrm{~h}$, cats were group-housed in the same room to allow for voluntary physical activity without human interference. The number of cats was 12,11

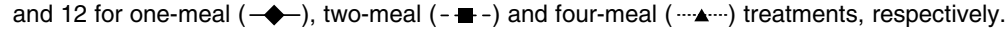

with the cats in the four times-daily-feeding group. Although it appears to be large and contains many toys, scratching posts, etc., the size of the animal room may also be a limiting factor in such a study.

Before the present study, cats were fed a commercial diet once daily at 08.00 hours. We used the same method and same housing schedule to measure the physical activity levels of all twelve cats when fed one meal daily. Due to the limitation of the study design, we feel it is not appropriate to perform statistical analysis on once daily feeding. However, it is interesting to note that the daily average activity level and the ratio of light-dark activities for one-meal-fed cats were numerically lower than the multiple-meal treatments. Slingerland et al. ${ }^{(2)}$ reported that physical inactivity and indoor confinement was significantly correlated with the development of type 2 diabetes mellitus in cats. In human subjects, reducing levels of physical activity can markedly affect energy balance. A sedentary routine does not induce decreased food intake but leads to a significantly positive energy balance, most of which has been stored as fat ${ }^{(7)}$. Research focusing on the influence of eating patterns on BW in male subjects suggested that eating frequency was negatively correlated with BW and body fatness ${ }^{(8,9)}$. It is unknown whether eating frequency affects BW by altering physical activity levels, hormone levels, metabolism or other unknown factors associated with energy homeostasis.

In the present study, one-meal and multiple-meal treatments resulted in different daily activity patterns. By contrast, two-meal and four-meal treatments resulted in very similar daily patterns. Activity levels peaked immediately before and during meal times or at times they anticipated a meal. The daily activity level of one-meal treatment peaked at the meal time (08.00 hours) and immediately after time in individual housing (14.00, 18.00 and 22.00 hours). This change of daily activity pattern may be related to the anticipation of a meal for cats, which may be influenced by cats' memories, surrounding cats, feeding behaviours of humans, or changes in appetite-regulating hormones or metabolism of a meal in cats. A recent mouse study has demonstrated that ghrelin was an important hormone coordinating behavioural activity rhythms with the timing of food availability. It acts in the hypothalamus to stimulate locomotor activity in anticipation of scheduled meals ${ }^{(10)}$. Ghrelin was not measured in the present study, but would be of interest in future studies.

In conclusion, there was no difference between two-time and four-time feeding patterns. However, multiple-meal feeding patterns may increase the activity of cats compared with one-meal feeding. Furthermore, study is needed to clarify the effects of feeding frequency on physical activity and the potential mechanisms involved.

\section{Acknowledgements}

The present study was supported by the USDA National Institute of Food and Agriculture, Hatch project no. ILLU-538-396. All authors state that there are no conflicts of interest. P. D. conducted the experimental work and wrote the manuscript. R. W. G. took part in the evaluation of the results. K. S. S. designed the experiment.

\section{References}

1. Lund EM, Armstrong PJ, Kirk CA, et al. (2005) Prevalence and risk factors for obesity in adult cats from private US veterinary practices. Intern J Appl Res Vet Med 3, 88-96.

2. Slingerland LI, Fazilova VV, Plantinga EA, et al. (2009) Indoor confinement and physical inactivity rather than the proportion of dry food are risk factors in the development of feline type 2 diabetes mellitus. Vet J 179, 247-253.

3. German A \& Martin L (2008) Epidemiology of obesity. In Feline Clinical Nutrition, pp. 26 [P Pibot, V Biourge and D Elliott, editors]. Aimargues-France: Aniwa SAS.

4. Belsito KR, Vester BM, Keel TL, et al. (2009) Impact of ovariohysterectomy and food intake on body composition, physical activity, and adipose gene expression in cats. J Anim Sci 87, 594-602. 
5. Vester BM, Liu KJ, Keel TL, et al. (2009) In utero and postnatal exposure to a high-protein or high-carbohydrate diet leads to differences in adipose tissue mRNA expression and blood metabolites in kittens. Br J Nutr 102, 1136-1144.

6. Association of American Feed Control Officials (2009) Official Publication of the Association of American Feed Control Officials. Oxford, IN: Association of American Feed Control Officials, Inc.

7. Stubbs RJ, Hughes DA, Johnstone AM, et al. (2004) A decrease in physical activity affects appetite, energy, and nutrient balance in lean men feeding ad libitum. Am J Clin Nutr 79, 62-69.
8. Drummond SE, Crombie NE, Cursiter MC, et al. (1998) Evidence that eating frequency is inversely related to body weight status in male, but not female, non-obese adults reporting valid dietary intakes. Int $J$ Obes Relat Metab Disord 22, 105-112.

9. Ruidavets JB, Bongard V, Bataille V, et al. (2002) Eating frequency and body fatness in middle-aged men. Int $J$ Obes 26, 1476-1483.

10. Blum ID, Patterson Z, Khazall R, et al. (2009) Reduced anticipatory locomotor responses to scheduled meals in ghrelin receptor deficient mice. Neuroscience $\mathbf{1 6 4}$, 351-359. 